\title{
Asiaticoside attenuates the effects of spinal cord injury through antioxidant and anti-inflammatory effects, and inhibition of the p38-MAPK mechanism
}

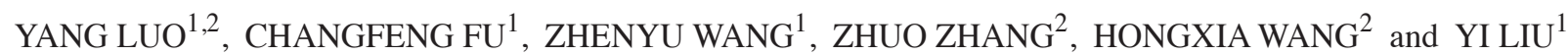 \\ ${ }^{1}$ Department of Spinal Surgery, First Hospital of Jilin University, Changchun, Jilin 130021; \\ ${ }^{2}$ Department of Orthopaedics, General Hospital of Chinese PLA, Beijing 100853, P.R. China
}

Received November 15, 2014; Accepted August 20, 2015

DOI: $10.3892 / \mathrm{mmr} .2015 .4425$

\begin{abstract}
Asiaticoside has potent pharmacological activity and broader pharmacological effects, including anti-oxidant, antidepressant and hepatic protection effects, and the inhibition of tumor cell proliferation. However, the mechanism underlying the effects of asiaticoside on neurological pain in spinal cord injury (SCI) remain to be fully elucidated. Therefore, the present study investigated the specific mechanism underlying the beneficial action of asiaticoside in a SCI rat model. In the present study, Basso, Beattie and Bresnahan scores was determined to analyze the therapeutic effects of asiaticoside on the neurological function of the SCI rat model. The water content of the spinal cord was also determined to measure its effects on the SCI rats. Oxidative stress, levels of nitric oxide and inflammation were detected using commercial kits. Western blot analysis was used to measure the protein expression levels of p38-mitogen-activated protein kinase (MAPK) in the SCI rat. Asiaticoside effectively augmented the Basso, Beattie and Bresnahan scores of the SCI rats. Significant reductions in the water content of the spinal cord, the levels of inducible nitric oxide synthase (iNOS), and the activities of the nuclear factor- $\kappa \mathrm{B}$ p65 unit, tumor necrosis factor- $\alpha$, interleukin(IL)-1 $\beta$ and IL-6 were observed in the experimental animals. Furthermore, on examination of the oxidative stress-associated parameters, increased production of malondialdehyde and decreased levels of superoxide dismutase, glutathione and glutathione peroxidase were detected in the SCI rat model. Asiaticoside also significantly suppressed the expression of p38-MAPK, which indicated that the therapeutic effects of asiaticoside may be associated with the p38-MAPK pathway. These data confirmed that asiaticoside attenuates
\end{abstract}

Correspondence to: Mr. Yi Liu, Department of Spinal Surgery, First Hospital of Jilin University, 71 Xinmin Street, Changchun, Jilin 130021, P.R. China

E-mail: yiliulxmm@163.com

Key words: asiaticoside, spinal cord injury, antioxidant, anti-inflammatory, p38-mitogen-activated protein kinase
SCI through antioxidant and anti-inflammatory effects, and through inhibition of the p38-MAPK mechanism.

\section{Introduction}

Spinal cord injury (SCI) is a serious complication of trauma often caused by car accidents, falls and other causes of spinal trauma, of which the symptoms include sensory and motor dysfunction below the damage plane, autonomic nerve dysfunction, difficulty in restoring nerve function following injury and high rates of morbidity $(1,2)$. The causes of these symptoms are predominantly secondary to violent injury, however, secondary SCI includes the integrity of blood-spinal cord barrier, neutrophil inflammatory cell infiltration following primary trauma, neuronal necrosis, apoptosis and glial scar formation (3). In previous years, the incidence of the disease has exhibited a gradually increasing trend, due to a lack of effective treatment options, and surgery cannot restore lost nerve functions (4).

The pathophysiological processes of SCI include the initial primary injury and the consequent secondary injury. Secondary injury is involved in a variety of molecular mechanisms, including $\mathrm{Ca}^{2+}$ influx overload, excitatory amino acid toxicity and oxidative stress (5). Oxidative stress directly damages the structure and the function of nerve cells by attacking biological macromolecules in the cells, and causes death of the nerve cells, which is closely associated with neurodegenerative disease following SCI (6). Therefore, antioxidative stress is an effective strategy for SCI therapeutic intervention. Cavus et al reported that montelukast and methylprednisolone have a neuroprotective effect on SCI by downregulating the levels of malondialdehyde (MDA), superoxide dismutase (SOD), glutathione peroxidase (GSH-PX) and catalase (7). Tavukçu et al (8) revealed that melatonin and tadalafil treatment improve erectile dysfunction following SCI through suppression of the levels of MDA, SOD and glutathione $(\mathrm{GSH})$ in rats.

The induction of secondary damage following SCI can lead to tissue hemorrhage, edema and apoptosis, and immune and inflammatory grade-linking reactions are further expanded, with inflammation being important in SCI. Early relief of inflammation following SCI is involved in neural protection 
and the promotion of functional recovery. Nuclear factor $(\mathrm{NF})-\kappa \mathrm{B}$, tumor necrosis factor (TNF)- $\alpha$ and interleukin (IL)-1 $\beta$ have been reported to be significantly downregulated following plumbagin treatment in SCI rats (9). Nacar et al (10) revealed the beneficial effect of atorvastatin in rat SCI through anti-inflammatory effects.

The mitogen-activated protein kinase (MAPK) family is a conservative group of serine/threonine protein kinase, and comprise a major group of signaling molecules in the transduction process, which is important in the development and occurrence of disease. MAPK activation is the final step in the intracellular phosphorylation cascade (11). P38 is a phosphoric acid protein tyrosine kinase, which is purified and isolated from mammalian cells stimulated by endotoxin. P38 is the most important member of the MAPK family in the control of the inflammatory response, which may be activated due to physiological stress, lipopolysaccharides, osmotic stress or ultraviolet irradiation (11). Galan-Arriero et al (12) reported that oral administration of an $\mathrm{p} 38 \alpha$ MAPK inhibitor inhibited affective pain behavior following SCI. Qu et al (13) also reported that inhibition of $\mathrm{p} 38$-MAPK signaling reduces the microglial inflammatory response in rats following SCI.

Asiaticoside is a white needle-like crystal, which is extracted from Centellaasiatica and has a swelling and detoxification effect, which is reported to have certain antitumor activities (14). It has been demonstrated that asiaticoside has potent pharmacological activity and broader pharmacological effects, having antioxidant, antidepressant and hepatic protective effects, and functioning in the inhibition of tumor cell proliferation. As these previous data presented only indirect evidence on the effects of asiaticoside on SCI, the present study aimed to investigate the mechanisms underlying the action of asiaticoside in neurological function using SCI model rats.

\section{Materials and methods}

Chemicals. Asiaticoside (purity $>95 \%$ ) was purchased from Nanjing Traditional Chinese Medicine Institute of Chinese Material Medica (Nanjing, China) and the chemical structure is indicated in Fig. 1. MPSS was purchased from the First Hospital of Jilin University (Changchun, China). MDA, SOD, GSH, GSH-PX, NF- $\kappa$ B p65 unit, TNF- $\alpha$, IL-1 $\beta$ and IL-6 commercial immunoassay kits were purchased from Elabscience Biotechnology Co., Ltd. (Wuhan, China). The Inducible Nitric Oxide Synthase (iNOS) commercial kit was purchased from Sangon Biotech Co., Ltd. (Shanghai, China).

Animals and ethical statement. The animals used in the present study were male Sprague-Dawley rats (8-10 weeks old; 250-280 g), which were obtained from the Animal Resource Center of the First Hospital of Jilin University and approved by the ethics committee of the First Hospital of Jilin University. The rats were individually housed in Plexiglas bins with food and water continuously available, and were maintained under a controlled environment at $23 \pm 1^{\circ} \mathrm{C}$ with a 12-h light/dark cycle and 60-80\% humidity. All experimental procedures were approved by the guidelines of the Care and Use of Laboratory Animals of the First Hospital of Jilin University.
Experimental groups and induction of the SCI rat model. The 50 male Sprague-Dawley rats were randomly assigned into five groups. The sham group $(n=10)$, received only physiological saline $(0.1 \mathrm{ml} / 100 \mathrm{~g}$, intraperitoneally). The remaining four groups underwent SCI at the T10 spinal segment impactor. The SCI group $(n=10)$ received no treatment following SCI, the ASI (30) group $(n=10)$ received asiaticoside at doses of $30 \mathrm{mg} / \mathrm{kg}$ once a day for 7 days following SCI (15), the ASI (60) group $(n=10)$ received asiaticoside at a dose of $60 \mathrm{mg} / \mathrm{kg}$ once a day for 7 days following SCI, the MPSS group $(n=10)$ received $100 \mathrm{mg} / \mathrm{kg}$ MPSS following SCI.

The rat model of SCI was induced, as previously described (16). The rats were anesthetized with an intraperitoneal injection of sodium pentobarbital $(50 \mathrm{mg} / \mathrm{kg}$; Sigma-Aldrich, St. Louis, MO, USA), and a laminectomy was performed at the T10 level to expose the cord beneath, without disrupting the dura. Subsequently, a rat model of spared cord injury was created by performing a laminectomy, during which the T8 and T9 vertebral peduncles were removed. The control model rats underwent the same laminectomy, but without compression.

Evaluation of neuronal function recovery. Following SCI, the evaluation of locomotor recovery was evaluated using the Basso, Beattie and Bresnahan (BBB), locomotor rating scale of $0-21$, in which 0 , indicated no observable hind-limb movements and 21 indicated normal locomotion (17).

Assessment of the water content of the spinal cord following SCI. At 72 h post-SCI, the spinal cord was collected, the wet weight was obtained and the spinal cord was placed in an oven at $80^{\circ} \mathrm{C}$ for $48 \mathrm{~h}$. The dry weight of the spinal cord was then measured. The plasma supernatant was collected following centrifugation at $5,000 \mathrm{x} \mathrm{g}$ for $10 \mathrm{~min}$ at $4^{\circ} \mathrm{C}$. The nitrite concentration was spectrophotometrically determined using a CM-2600d spectrophotometer (Konica Minolta Sensing Singapore Pte Ltd., Jurong East, Singapore) and the following formula: Spinal cord water content $(\%)=($ wet weight - dry weight $) /$ wet weight $\times 100 \%$.

Measurement of levels of MDA, SOD, GSH and GSH-PX. Following treatment with asiaticoside for seven consecutive days, the peripheral blood was collected from each group. Subsequently, the supernatant was centrifuged at $12,000 \mathrm{x} \mathrm{g}$ for $20 \mathrm{~min}$ at $4^{\circ} \mathrm{C}$. The serum levels of MDA, SOD, GSH and GSH-PX were analyzed using commercial immunoassay kits (cat. nos. E-EL-0060c, E-EL-R1424c, E-EL-R0440c and E-EL-R2491c, respectively), according to the manufacturer's instructions (Elabscience Biotechnology Co., Ltd.

Measurement of iNOS activity. Following treatment with asiaticoside for seven consecutive days, the rats were sacrificed via cervical dislocation and spinal cord tissue was collected from each group. The activity of iNOS was determined using a commercial kit, according to the manufacturer's instructions (Sangon Biotech Co., Ltd.).

Measurement of the activities of NF- $\kappa B$ p 65 unit, TNF- $\alpha, I L-1 \beta$ and $I L-6$. Following treatment with asiaticoside for seven consecutive days, the peripheral blood was collected from 


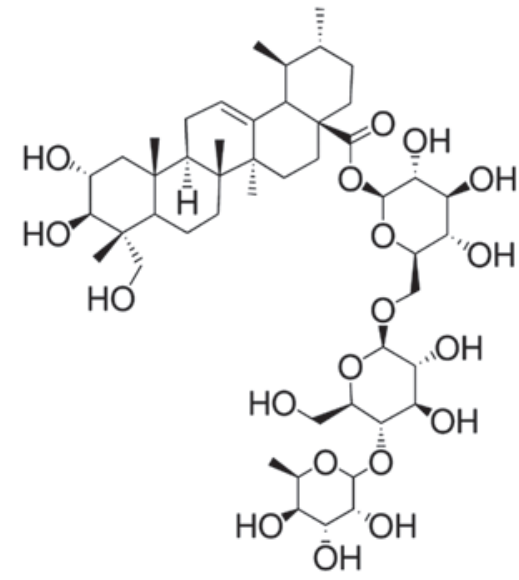

Figure 1. Chemical structure of asiaticoside.

each group. Subsequently, the supernatants were centrifuged at $12,000 \mathrm{xg}$ for $20 \mathrm{~min}$ at $4^{\circ} \mathrm{C}$. The serum activities of NF- $\mathrm{\kappa B}$ p65 unit, TNF- $\alpha$, IL-1 $\beta$ and IL-6 (cat. nos. E-EL-R0674c, E-CL-R0019c, E-EL-R0012c and E-EL-R0015c, respectively) were analyzed using the respective commercial immunoassay kits, according to the manufacturer's instructions (Elabscience Biotechnology Co., Ltd.).

Western blot analysis. Samples ( 10 mg) of the exposed spinal cord tissue were removed from the rats and incubated with $100 \mu 1$ ice-cold lysis buffer, containing $2 \mathrm{mM}$ EDTA, $10 \mathrm{mM}$ EGTA, $0.4 \% \mathrm{NaF}, 20 \mathrm{mM}$ Tris- $\mathrm{HCl}$ and protease inhibitors ( $\mathrm{pH} 7.5$ ) for 10-15 min on ice. Subsequently, the homogenates were centrifuged at $12,000 \mathrm{x}$ g for $20 \mathrm{~min}$ at $4^{\circ} \mathrm{C}$. The protein concentrations of the soluble materials were determined using a Bicinchoninic Acid protein assay (Beyotime Institute of Biotechnology, Nanjing, China). Equal quantities of protein $(30 \mu \mathrm{g})$ were separated on $12 \%$ sodium dodecyl sulfate-polyacrylamide gels (Beyotime Institute of Biotechnology), followed by transfer onto polyvinylidene fluoride membranes $(0.22 \mathrm{~mm}$; EMD Millipore, Billerica, MA, USA). The membranes were blocked with phosphate-buffered saline with 5\% non-fat milk to block nonspecific binding sites. Subsequently, the membranes were incubated with monoclonal mouse anti-human anti-p38-MAPK (cat. no. sc-4708 1:2,000; Santa Cruz Biotechnology, Inc., Santa Cruz, CA, USA) and monoclonal anti- $\beta$-actin (cat. no. D110001; 1:500; Sangon Biotech Co., Ltd.) overnight at $4^{\circ} \mathrm{C}$. The membrane was then washed three times with Tris-buffered saline with Tween 20 (Senbeijia Biotech Co., Nanjing, China) for $2 \mathrm{~h}$, and then detected by incubation with anti-mouse IgG (cat. no. sc-358922; 1:1,000; Santa Cruz Biotechnology, Inc.) conjugated with horseradish peroxidase for $2 \mathrm{~h}$ at room temperature. The relative band intensity was determined using a gel image analysis system (Pierce Biotechnology, Inc., Rockford, IL, USA).

Statistical analysis. All the data were analyzed using SPSS version 17.0 (SPSS, Inc., Chicago, IL, USA) and expressed as the mean \pm standard deviation. Statistical analysis was performed using one-way analysis of variance, followed by Dunnett's test. $\mathrm{P}<0.05$ was considered to indicate a statistically significant difference.

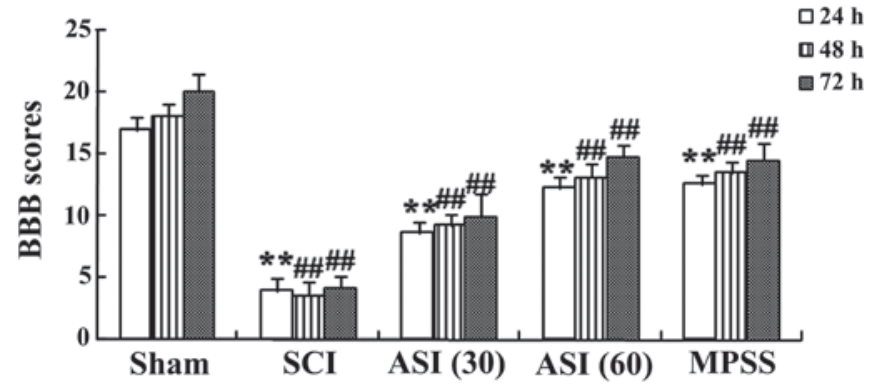

Figure 2. Effects of asiaticoside on neurological function following SCI. Data are expressed as the mean \pm standard deviation. ${ }^{* *} \mathrm{P}<0.01$, compared with the control group; ${ }^{\# /} \mathrm{P}<0.01$, compared with the SCI group. Con, control; SCI, spinal cord injury; ASI (30), asiaticoside (30 mg/kg); ASI (60), asiaticoside $(60 \mathrm{mg} / \mathrm{kg})$; MPSS, methylprednisolone; BBB, Basso, Beattie and Bresnahan.

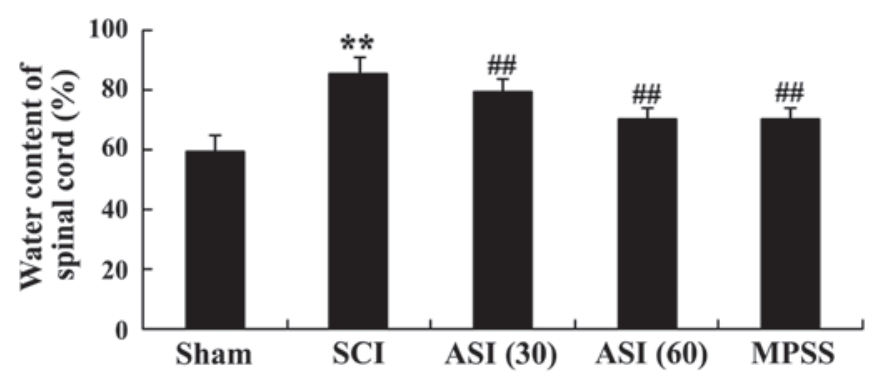

Figure 3. Effects of asiaticoside on the water content of the spinal cord following SCI. Data are expressed as the mean \pm standard deviation. ${ }^{* *} \mathrm{P}<0.01$, compared with the control group; ${ }^{\#} \mathrm{P}<0.01$, compared with the SCI group. Con, control; SCI, spinal cord injury; ASI (30), asiaticoside (30 mg/kg); ASI (60), asiaticoside (60 mg/kg); MPSS, methylprednisolone.

\section{Results}

Effects of asiaticoside on neurological function. In the present study, BBB scores were used to assess neurological function at $24 \mathrm{~h}, 48 \mathrm{~h}$ and $72 \mathrm{~h}$ following SCI, the results of which are presented in Fig. 2. The mean BBB scores of the SCI group were lower than the sham-operated group. However, it was noted that the injured rats of the asiaticoside-treated group had particularly high BBB scores. As shown in Fig. 2, the BBB scores following treatment with asiaticoside at a dose of $60 \mathrm{mg} / \mathrm{kg}$ were similar to those of the MPSS group $(\mathrm{P}>0.05)$.

Effects of asiaticoside on the water content of the spinal cord following SCI. The rats induced by SCI exhibited severe impairment with marked increase in water content of the spinal cord following SCI (Fig. 3). However, treatment with asiaticoside at different doses ( 30 and $60 \mathrm{mg} / \mathrm{kg}$ ) of the injured rats significantly reduced the water content of the spinal cord, compared with the SCI model group (Fig. 3). No significant difference was observed between the MPSS group and the $60 \mathrm{mg} / \mathrm{kg}$ asiaticoside group $(\mathrm{P}>0.05)$.

Anti-oxidative effects of asiaticoside. It was previously reported that serum cytokine levels are relevant to SCI (18). Therefore, the serum levels of oxidant stress were determined in the present study. As shown in Fig. 4A, the MDA concentrations of the SCI group were higher than those in the sham-operated group. In the asiaticoside-treated group, 
A

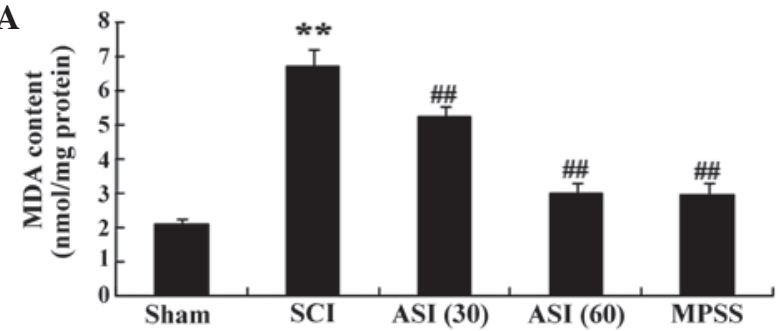

C

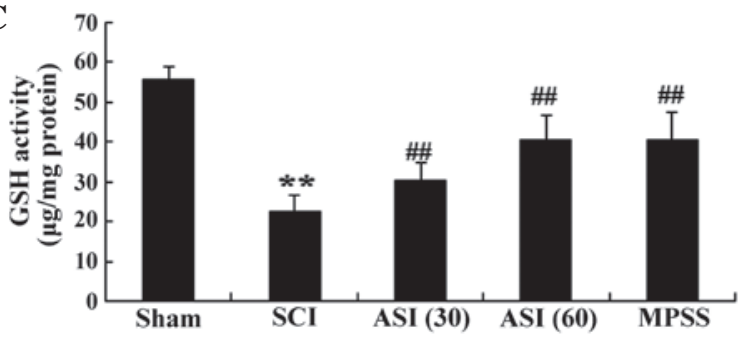

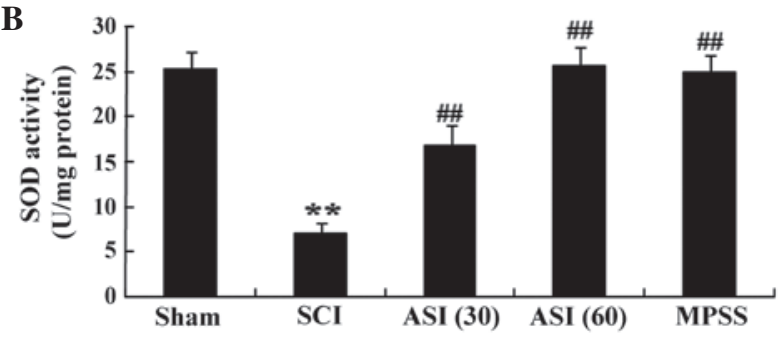

D

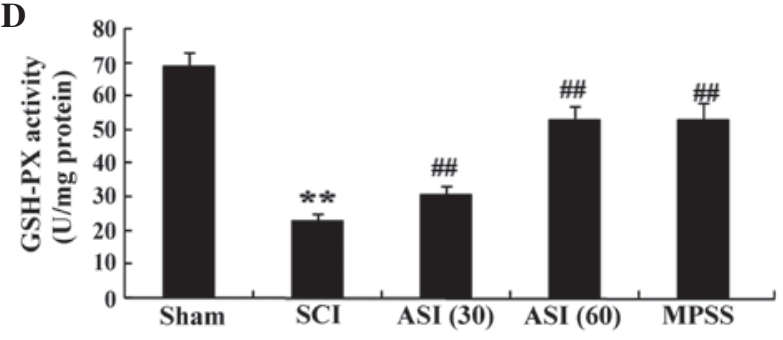

Figure 4. Anti-oxidative effects of asiaticoside following SCI. Anti-oxidative effects of asiaticoside on the concentrations of (A) MDA, (B) SOD, (C) GSH and (D) GSH-PX in the SCI model rats. Data are expressed as the mean \pm standard deviation. ${ }^{* *} \mathrm{P}<0.01$, compared with the control group; ${ }^{\# /} \mathrm{P}<0.01$, compared with the SCI group. Con, control; SCI, spinal cord injury; ASI (30), asiaticoside (30 mg/kg); ASI (60), asiaticoside (60 mg/kg); MPSS, methylprednisolone; MDA, malondialdehyde; SOD, superoxide dismutase; GHS, glutathione; GSH-PX, glutathione peroxidase.

the serum levels of MDA were also lower than those in the SCI group (Fig. 4A). The levels of SOD, GSH and GSH-PX in the SCI group were lower than those of the sham-operated group (Fig. 4B-D). The expression levels of SOD, GSH and GSH-PX in the asiaticoside-treated group were gradually increased, compared with those of the SCI group. However, no significant changes in cytokine levels between the MPSS group and $60 \mathrm{mg} / \mathrm{kg}$ asiaticoside treatment group were observed (Fig. 4A-D).

Anti-oxidative effects of asiaticoside on iNOS. The present study further investigated whether asiaticoside exerted protection against SCI through the mediation of nitric oxide. As shown in Fig. 5, iNOS activity was markedly increased in the spinal cord tissues of the SCI group. However, administration with asiaticoside (30 and $60 \mathrm{mg} / \mathrm{kg}$ ) generated a more pronounced reduction in iNOS activity in the SCI-induced rats (Fig. 5). In addition, the anti-oxidative effect of asiaticoside at a dose of $60 \mathrm{mg} / \mathrm{kg}$ was equipotent to that of the MPSS group (Fig. 5).

Anti-inflammatory effects of asiaticoside. The present study used ELISA commercial immunoassay kits to determine the expression levels of inflammatory factors, for assessment of the progression of the SCI. There were increases in the serum levels of the NF- $\kappa$ B p 65 unit, TNF- $\alpha$, IL- $1 \beta$ and IL- 6 in the SCI group, compared with the sham group (Fig. 6A-D). However, asiaticoside treatment of the SCI-induced rats reversed these indices (Fig. 6A-D). The anti-inflammatory effect of asiaticoside $(60 \mathrm{mg} / \mathrm{kg})$ was equipotent to that in the MPSS group (Fig. 6A-D).

Astaxanthin adjusts the expression of p38-MAPK. The present study further investigated whether asiaticoside exerted protection against SCI through mediation of the expression of p38-MAPK. As shown in Fig. 7A, western blot analysis using the p38-MAPK antibody demonstrated the anticipated bands

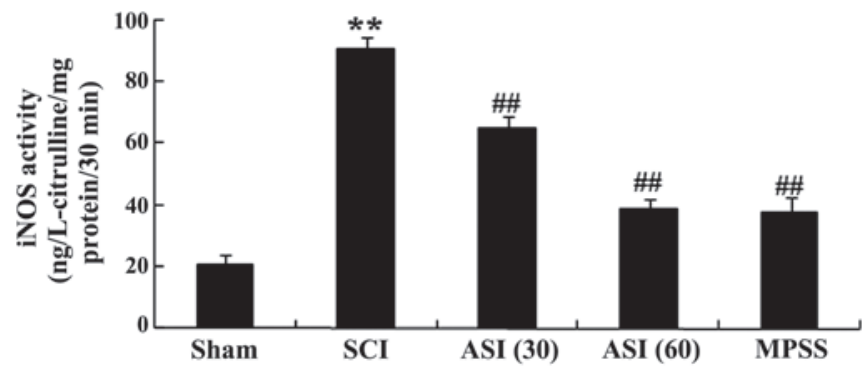

Figure 5. Anti-oxidative effects of asiaticoside on iNOS. Data are expressed as the mean \pm standard deviation. ${ }^{* *} \mathrm{P}<0.01$, compared with the control group; ${ }^{\# \#} \mathrm{P}<0.01$, compared with the SCI group. Con, control; SCI, spinal cord injury; ASI (30), asiaticoside (30 mg/kg); ASI (60), asiaticoside (60 mg/kg); MPSS, methylprednisolone; iNOS, inducible nitric oxide synthase.

of $43 \mathrm{kDa}$. Quantitative analysis disclosed an evident elevation of p38-MAPK protein in the SCI group, compared with the sham group (Fig. 7B). However, asiaticoside treatment (30 and $60 \mathrm{mg} / \mathrm{kg}$ ) markedly decreased the protein expression of p38-MAPK, compared with the SCI group (Fig. 7A-B). No significant inter-group differences were observed between the MPSS group and asiaticoside treatment $(60 \mathrm{mg} / \mathrm{kg})$ group in the protein expression of $\mathrm{p} 38$-MAPK in the SCI model rat (Fig. 7A-B).

\section{Discussion}

SCI is a trauma-induced disease, the causes of which predominantly include injury from car accidents and falls from heights (18). The treatment of SCI is limited, and the majority of patients have various degrees of sensory and motor nerve dysfunction, autonomic dysfunction, a reduction or loss of self-care ability and difficulty recovering, significantly affecting quality of life and introducing a serious burden for individuals, families and society (19). In the present study, it was first demonstrated that asiaticoside increased the BBB 
A
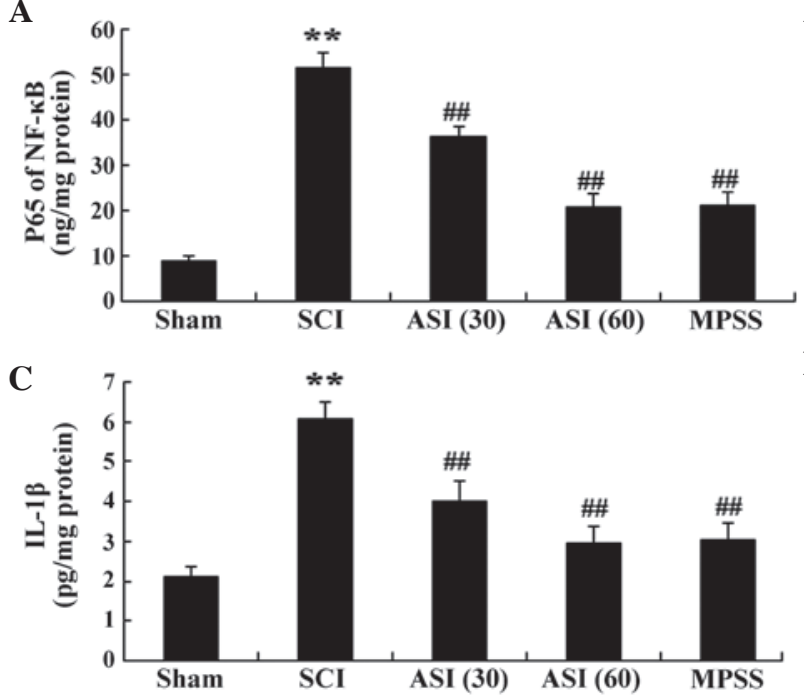

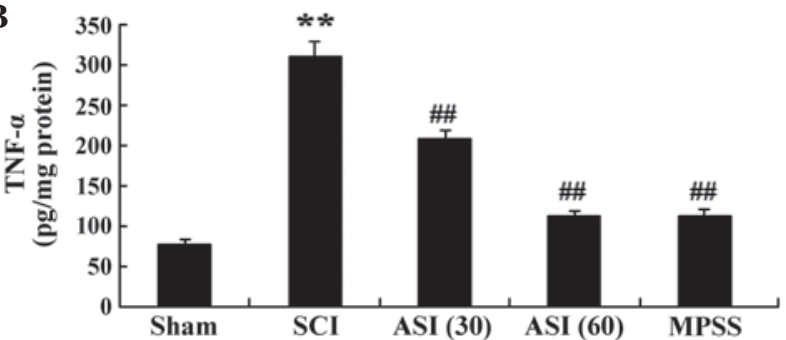

D

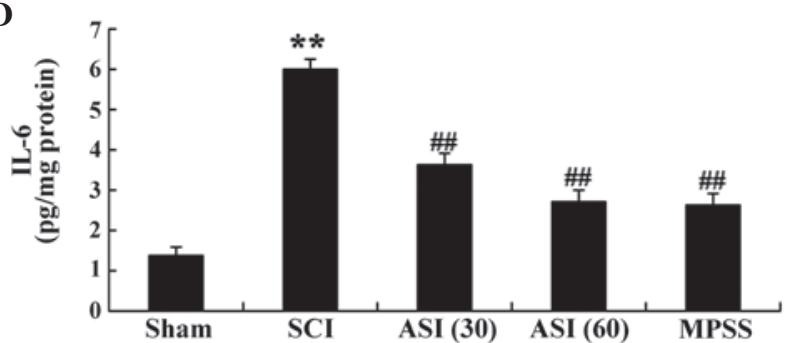

Figure 6. Anti-inflammatory effects of asiaticoside following SCI. Anti-inflammatory effects of asiaticoside on the serum activities of (A) NF- $\mathrm{B}$ p65, (B) TNF- $\alpha$ (B), (C) IL-1 $\beta$ (C) and (D) IL-6 in the SCI model rats. Data are expressed as the mean \pm standard deviation. ${ }^{* *} \mathrm{P}<0.01$, compared with the control group; ${ }^{\# \#} \mathrm{P}<0.01$, compared with the SCI group. Con, control; SCI, spinal cord injury; ASI (30), asiaticoside (30 mg/kg); ASI (60), asiaticoside (60 mg/kg); MPSS, methylprednisolone; IL, interleukin.
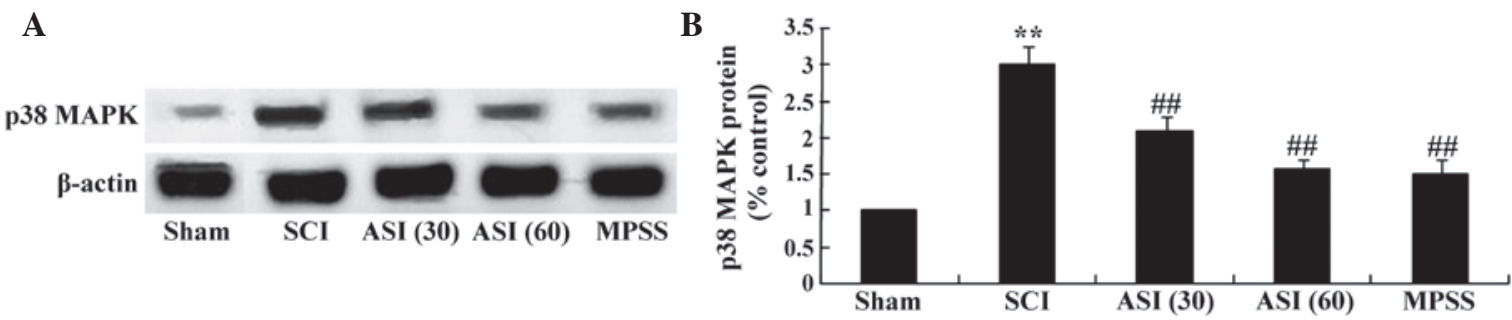

Figure 7. Astaxanthin alters the expression of the p38-MAPK pathway. (A) Effects of asiaticoside on the expression of p38-MAPK were determined using western blot analysis. (B) Statistical analysis of the protein levels of p38-MAPK in the SCI model rats. Data are expressed as the mean \pm standard deviation. ${ }^{* *} \mathrm{P}<0.01$, compared with the control group; ${ }^{\# /} \mathrm{P}<0.01$, compared with the SCI group. Con, control; SCI, spinal cord injury; ASI (30), asiaticoside (30 mg/kg); ASI (60), asiaticoside (60 mg/kg); MPSS, methylprednisolone; MAPK, mitogen-activated protein kinase.

scores and reduced the water content of the spinal cord following SCI. No statistically significant differences were identified between the asiaticoside $(60 \mathrm{mg} / \mathrm{kg})$ and MPSS groups.

Currently, it is considered that a lot of oxygen free radicals (OFRs) are generated in SCI pathogenesis. OFR damage to the body may act with a trigger-like effect, with calcium overload being a final common pathway for cell damage (20). SOD is an enzyme, which catalyzes disproportionation of superoxide anions, as a major intracellular antioxidant enzyme and free radical scavenger, which can protect cells against oxygen free radicals, and the level of which indicates the strength of the effectiveness in protecting cells from toxic oxygen free radical damage (21). MDA is the end product of lipid peroxidation, and can be measured to directly reflect the level of free radicals and is an important indicator of the level of tissue injury (22). Measuring the activities of MDA and SOD can indirectly reflect the antioxidant abilities of the body (23). In the present study, it was demonstrated that asiaticoside reduced the activities of MDA and iNOS and induced the levels of SOD, GSH and GSH-PX in the SCI rats. The anti-oxidative effects of asiaticoside induce the levels of SOD, GSH and GSH-PX in healing wounds (24), and the anti-inflammatory effects of asiaticoside dependently inhibit liver myeloperoxidase (MPO) activity and the protein expression of brain cyclooxygenase-2 (COX-2) (25). Xu et al (26) indicated that asiaticoside is effective in reversing 1-methyl-4-phenyl-1,2,3,6-tetrahydropyridine-induced Parkinsonism through the levels of MDA and GSH, whereas Guo et al (27) indicated that the inhibitory effects of asiaticoside in gastric ulcer healing in rats via the inhibition of iNOS.

The SCI process is associated with the production and release of inflammatory mediators, microvascular endothelial function disorder, inflammatory cell infiltration and accumulation in the spinal cord tissue, and increased expression of inflammatory cytokines and adhesion molecules (16). The combined effect of these factors trigger a cascade of inflammation, thereby increasing secondary injury in the ischemic area (28). Avascular necrosis of neurons, glial cells and endothelial cells following SCI can induce the production of large quantities of inflammatory cytokines, including TNF- $\alpha$, IL-1 $\beta$ and IL-6, which can stimulate the production of cytokines and other inflammatory mediators, affecting gene expression in glial cells (29). These cytokines can be used as a signaling molecule of endothelial cell activation, thereby stimulating the secretion of cell adhesion molecules and the adhesion between 
leukocytes and endothelial cells, which induce the infiltration of leukocytes to the damage zone (30). Leukocyte infiltration damages the blood brain barrier, further aggravating SCI. Studies have demonstrated that there are several factors involved in the process of apoptosis following SCI, in which inflammatory cytokines are important. The present study demonstrated decreased activities of the NF- $\kappa \mathrm{B}$ p65 Unit, TNF- $\alpha$, IL-1 $\beta$ and IL- 6 in SCI rats. Zhang et al (31) suggested that the protective effects of asiaticoside effectively protected against septic lung injury through the regulation of the iNOS, TNF- $\alpha$, IL- 6 and NF- $\kappa$ B pathway. Bhaumik et al (32) reported that asiaticoside induces $\mathrm{TNF}-\alpha$ to treat experimental visceral leishmaniasis via nitric oxide production.

The P38-MAPK signaling pathway is one of the three classical branches of the MAPK signaling pathway, widely involved in stress responses, including inflammation and radioactive injury. Studies have reported that activation of the p38-MAPK pathway enables the expression of downstream MAPK (MK)2 to be increased, promoting the expression of MMP-9 following SCI, leading to destruction of the blood-spinal cord barrier (33-35). The findings of the present study provide the first direct evidence, to the best of our knowledge, that asiaticoside restrained the protein expression of p38-MAPK in SCI rats. Chen et al (15) suggested that asiaticoside attenuates memory impairment through anti-inflammatory effects and inhibiting the overactivation of the p38-MAPK pathway. Zhang et al (36) reported that the protective effects of asiaticoside on acute liver injury are induced by restricting TNF- $\alpha$ and the p38-MAPK pathway in mice.

In conclusion, the major finding of the present study was that asiaticoside successfully decreased water content in SCI rats. Asiaticoside appeared to inhibit oxidative damage, nitric oxide activity, pro-inflammatory cytokine production and the p38-MAPK pathway. Further investigations on the signaling pathways and cross-talk consequent to asiaticoside administration may provide further insights into its therapeutic action in terms of SCI, and provide a starting point for developing novel strategies for pain control.

\section{References}

1. Liu C, Wu W, Zhang B, Xiang J and Zou J: Temporospatial expression and cellular localization of glutamine synthetase following traumatic spinal cord injury in adult rats. Mol Med Rep 7: 1431-1436, 2013.

2. Ravikumar R, Fugaccia I, Scheff SW, Geddes JW, Srinivasan C and Toborek M: Nicotine attenuates morphological deficits in a contusion model of spinal cord injury. J Neurotrauma 22: 240-251, 2005

3. Furlan JC, Sakakibara BM, Miller WC and Krassioukov AV: Global incidence and prevalence of traumatic spinal cord injury. Can J Neurol Sci 40: 456-464, 2013.

4. Hong Z, Hong H, Chen H, Wang Z and Hong D: Investigation of the protective effect of erythropoietin on spinal cord injury in rats. Exp Ther Med 2: 837-841, 2011.

5. Oyinbo CA: Secondary injury mechanisms in traumatic spinal cord injury: A nugget of this multiply cascade. Acta Neurobiol Exp (Wars) 71: 281-299, 2011.

6. Smith JA, Park S, Krause JS and Banik NL: Oxidative stress, DNA damage and the telomeric complex as therapeutic targets in acute neurodegeneration. Neurochem Int 62: 764-775, 2013.

7. Cavus G, Altas M, Aras M, Ozgür T, Serarslan Y, Yilmaz N, Sefil F and Ulutas KT: Effects of montelukast and methylprednisolone on experimental spinal cord injury in rats. Eur Rev Med Pharmacol Sci 18: 1770-1777, 2014.
8. Tavukçu HH, Sener TE, Tinay I, Akbal C, Erşahin M, Cevik O, Cadirci S, Reiter RJ and Sener G: Melatonin and tadalafil treatment improves erectile dysfunction after spinal cord injury in rats. Clin Exp Pharmacol Physiol 41: 309-316, 2014.

9. Zhang W, Cheng L, Hou Y, Si M, Zhao YP and Nie L: Plumbagin protects against spinal cord injury-induced oxidative stress and inflammation in wistar rats through Nrf-2 upregulation. Drug Res (Stuttg) 2014.

10. Nacar OA, Eroglu H, Cetinalp NE, Menekse G, Yildirim AE, Uckun OM, Daglioglu E, Turkoglu OF and Belen AD: Systemic administration of atorvastatin improves locomotor functions and hyperacute-acute response after experimental spinal cord injury: An ultrastructural and biochemical analysis. Turk Neurosurg 24: 337-343, 2014.

11. Kanno A, Ozawa T and Umezawa Y: Bioluminescent imaging of MAPK function with intein-mediated reporter gene assay. Methods Mol Biol 574: 185-192, 2009.

12. Galan-Arriero I, Avila-Martin G, Ferrer-Donato A, Gomez-Soriano J, Bravo-Esteban E and Taylor J: Oral administration of the p38 $\alpha$ MAPK inhibitor, UR13870, inhibits affective pain behavior after spinal cord injury. Pain 155: 2188-2198, 2014

13. Qu WS, Tian DS, Guo ZB, Fang J, Zhang Q, Yu ZY, Xie MJ, Zhang HQ, Lü JG and Wang W: Inhibition of EGFR/MAPK signaling reduces microglial inflammatory response and the associated secondary damage in rats after spinal cord injury. J Neuroinflammation 9: 178, 2012.

14. Lin X, Huang R, Zhang S, Wei L, Zhuo L, Wu X, Tang A and Huang Q: Beneficial effects of asiaticoside on cognitive deficits in senescence-accelerated mice. Fitoterapia 87: 69-77, 2013.

15. Chen S, Yin ZJ, Jiang C, Ma ZQ, Fu Q, Qu R and Ma SP: Asiaticoside attenuates memory impairment induced by transient cerebral ischemia-reperfusion in mice through anti-inflammatory mechanism. Pharmacol Biochem Behav 122: $7-15,2014$.

16. Hsu JY, McKeon R, Goussev S, Werb Z, Lee JU, Trivedi A and Noble-Haeusslein LJ: Matrix metalloproteinase-2 facilitates wound healing events that promote functional recovery after spinal cord injury. J Neurosci 26: 9841-9850, 2006

17. Basso DM, Beattie MS, Bresnahan JC, Anderson DK, Faden AI, Gruner JA, Holford TR, Hsu CY, Noble LJ, Nockels R, et al: MASCIS evaluation of open field locomotor scores: Effects of experience and teamwork on reliability. ++multicenter animal spinal cord injury study. J Neurotrauma 13: 343-359, 1996.

18. Wang Z, Zhang C, Hong Z, Chen H, Chen W and Chen G: C/ EBP homologous protein (CHOP) mediates neuronal apoptosis in rats with spinal cord injury. Exp Ther Med 5: 107-111, 2013.

19. Liu G, Wang X, Shao G and Liu Q: Genetically modified Schwann cells producing glial cell line-derived neurotrophic factor inhibit neuronal apoptosis in rat spinal cord injury. Mol Med Rep 9: 1305-1312, 2014.

20. Xie YG, Mu HJ, Li Z, Ma JH and Wang YL: Supression of chronic central pain by superoxide dismutase in rats with spinal cord injury: Inhibition of the NMDA receptor implicated. Exp Ther Med 8: 1137-1141, 2014.

21. Kurtoglu T, Basoglu H, Ozkisacik EA, Cetin NK, Tataroglu C, Yenisey C and Discigil B: Effects of cilostazol on oxidative stress, systemic cytokine release, and spinal cord injury in a rat model of transient aortic occlusion. Ann Vasc Surg 28: 479-488, 2014

22. Li YD, Ma YH, Zhao JX and Zhao XK: Protection of ultra-filtration extract from Danggui Buxue Decoction on oxidative damage in cardiomyocytes of neonatal rats and its mechanism. Chin J Integr Med 17: 854-859, 2011.

23. Song Y, Liu J, Zhang F, Zhang J, Shi T and Zeng Z: Antioxidant effect of quercetin against acute spinal cord injury in rats and its correlation with the p38MAPK/iNOS signaling pathway. Life Sci 92: 1215-1221, 2013.

24. Shukla A, Rasik AM and Dhawan BN: Asiaticoside-induced elevation of antioxidant levels in healing wounds. Phytother Res 13: 50-54, 1999.

25. Wan J, Gong X, Jiang R, Zhang Z and Zhang L: Antipyretic and anti-inflammatory effects of asiaticoside in lipopolysaccharide-treated rat through up-regulation of heme oxygenase-1. Phytother Res 27: 1136-1142, 2013.

26. Xu CL, Wang QZ, Sun LM, Li XM, Deng JM, Li LF, Zhang J, $\mathrm{Xu} \mathrm{R}$ and Ma SP: Asiaticoside: Attenuation of neurotoxicity induced by MPTP in a rat model of Parkinsonism via maintaining redox balance and up-regulating the ratio of $\mathrm{Bcl}-2 / \mathrm{Bax}$. Pharmacol Biochem Behav 100: 413-418, 2012. 
27. Guo JS, Cheng CL and Koo MW: Inhibitory effects of Centella asiatica water extract and asiaticoside on inducible nitric oxide synthase during gastric ulcer healing in rats. Planta Med 70: $1150-1154,2004$.

28. Manhas A, Khanna V, Prakash P, Goyal D, Malasoni R, Naqvi A, Dwivedi AK, Dikshit M and Jagavelu K: Curcuma oil reduces endothelial cell-mediated inflammation in postmyocardial ischemia/reperfusion in rats. J Cardiovasc Pharmacol 64: 228-236, 2014

29. Nguyen DH, Cho N, Satkunendrarajah K, Austin JW, Wang J and Fehlings MG: Immunoglobulin $\mathrm{G}$ ( $\mathrm{IgG}$ ) attenuates neuroinflammation and improves neurobehavioral recovery after cervical spinal cord injury. J Neuroinflammation 9: 224, 2012.

30. Casella GT, Bunge MB and Wood PM: Improved immunocytochemical identification of neural, endothelial, and inflammatory cell types in paraffin-embedded injured adult rat spinal cord. J Neurosci Methods 139: 1-11, 2004.

31. Zhang LN, Zheng JJ, Zhang L, Gong X, Huang H, Wang CD, Wang B, Wu MJ, Li XH, Sun WJ, et al: Protective effects of asiaticoside on septic lung injury in mice. Exp Toxicol Pathol 63: $519-525,2011$
32. Bhaumik SK, Paul J,Naskar K, Karmakar S and De T: Asiaticoside induces tumour-necrosis-factor- $\alpha$-mediated nitric oxide production to cure experimental visceral leishmaniasis caused by antimony-susceptible and -resistant Leishmania donovani strains. J Antimicrob Chemother 67: 910-920, 2012.

33. Zhou C, Shi X, Huang H, Zhu Y and Wu Y: Montelukast attenuates neuropathic pain through inhibiting p38 mitogen-activated protein kinase and nuclear factor-kappa $B$ in a rat model of chronic constriction injury. Anesth Analg 118: 1090-1096, 2014.

34. Ghasemlou N, Lopez-Vales R, Lachance C, Thuraisingam T, Gaestel M, Radzioch D and David S: Mitogen-activated protein kinase-activated protein kinase 2 (MK2) contributes to secondary damage after spinal cord injury. J Neurosci 30: 13750-13759, 2010.

35. Li XQ, Cao XZ, Wang J, Fang B, Tan WF and Ma H: Sevoflurane preconditioning ameliorates neuronal deficits by inhibiting microglial MMP-9 expression after spinal cord ischemia/reperfusion in rats. Mol Brain 7: 69, 2014

36. Zhang L, Li HZ, Gong X, Luo FL, Wang B, Hu N, Wang CD, Zhang $\mathrm{Z}$ and Wan JY: Protective effects of Asiaticoside on acute liver injury induced by lipopolysaccharide/D-galactosamine in mice. Phytomedicine 17: 811-819, 2010. 